\title{
Correspondence
}

\section{The State of the World's Children}

Sir,

We were interested to read in David Morley's recent annotation 'The State of the World's Children' that in the Gambia there is a $10 \%$ mortality in the subsequent nine months after infection with measles, and this compares with only $1 \%$ in controls. ${ }^{1}$

Our own interest in the possible predisposition to subsequent death from measles infection was aroused by two deaths within 24 hours from meningococcaemia, which occurred in this hospital earlier this year. Both children were girls aged 2 years, and one had had measles eight weeks before her death and the other six weeks before.

Meningococcal infection is an important cause of morbidity and mortality in childhood, causing $2 \%$ of all deaths between 1 and 4 years in the United Kingdom. It has been suggested that host factors-for example, familial complement deficiency ${ }^{2}$ - may be operative in some cases of fulminating disease, but in most cases no such factors are recognised and immunosuppression is a well known associate with measles. Niwa et al have recently shown that suppressor $T$ lymphocytes from patients with measles can significantly depress oxygen radical generation by normal polymorphs. ${ }^{3}$ This effect was shown to last for two months and was more pronounced in patients with secondary bacterial complications. There has been speculation that other viral infections may predispose to infection with Neisseria meningitidis as Young et al reported a simultaneous outbreak of influenza and meningococcal infection in Mississippi where there was a significant association between serologic evidence of influenza and systemic infection with or nasopharyngeal carriage of Neisseria meningitidis. ${ }^{4}$

The figures from the Gambia would seem to support further the essential inclusion of measles immunisation in any population immunisation programme, and we would be interested in any other instances of fatal meningococcal infection with preceding measles, because although both measles and meningococcal infection are both common in children, particularly in the first years of life, demonstration of a possible association between the two would provide further support for an effort to promote measles immunisation in the United Kingdom.

\section{J A Sills, D J Manning, and C A Hart Alder Hey Children's Hospital, Liverpool $L 122 A P$}

${ }^{3}$ Niwa Y, Sakane T, Somiya K, et al. Decreased oxygen radical generation by neutrophils from patients with measles, presumably owing to activation of suppressor $\mathrm{T}$ lymphocytes. $\mathrm{J}$ Clin Microbiol 1985;21:318-22.

4 Young LS, La Force FM, Head JJ, et al. A simultaneous outbreak of meningococcal and influenzal infections. $N$ Engl J Med 1972;287:5-9.

\section{Small bowel biopsy}

Sir,

I read with interest the Personal practice concerning small bowel biopsy of Dr Collins and colleagues. ${ }^{1}$ I would concur that semi-rigid catheters are superior to the very soft tubing supplied with some capsules. Indeed we have found that metal braided angiocardiographic catheters are superior to KIFA catheter tubing, which does not satisfactorily transmit torque and has a tendency to coil in the stomach. We have also found it helpful to inject some air into the stomach through the capsule and lie the child on the right side so that the capsule falls into the antral region.

In view of the extrapyramidal signs sometimes associated with metoclopramide, perhaps this should not be used routinely?

DONALD SHaw

Queen Elizabeth Hospital, London WIM $7 A B$

Drs Rolles and Collins comment:

We agree with Dr Shaw that the rigidity of the capsule tubing is of major importance and shall try the catheters he suggests with interest. As mentioned in our article, we always lie the child on the right side and have also found the injection of air to be useful if there is delay in the capsule passing through the pylorus.

With a combined experience of around 1500 biopsies we have never had difficulties with the use of metoclopramide as described. On those occasions when it was omitted or when intubation was unduly delayed after giving the metoclopramide the biopsy procedure was prolonged.

\section{Reference}

${ }^{1}$ Collins AL, Brookfield DSK, Hyde I, Rolles CJ. Small bowel biopsy. Arch Dis Child 1985;60:1082-5.

\section{Early congenital syphilis still occurs}

Sir,

We read with interest the article on the presentation of early congenital syphilis by Ewing et al. ${ }^{1}$ Of the seven 